\title{
Eventos Comunicativos de Esperanza en el Contexto de Educación Ambiental en la Formación Inicial de Profesores en Colombia.
}

\section{Communication Events of Hope in the Context of Environmental Education in Initial Teacher Training in Colombia.}

\author{
María Angélica Mejía-Cáceres ${ }^{1}$; Laísa Maria Freire ${ }^{2}$
}

1 Doutora em Educação em Ciências e Saúde, Programa de Pós-graduação em Educação em Ciências e Saúde, Universidade Federal do Rio de Janeiro, Rio de Janeiro, Brasil, missangelux @ hotmail.com, https://orcid.org/0000-0003-3486-1952

2 Doutora em Educação em Ciências e Saúde, Programa de Pós-graduação em Educação em Ciências e Saúde Programa de Pós-graduação em Ciências Ambientais e Conservação, Instituto de Biologia, Universidade Federal do Rio de Janeiro, Rio de Janeiro, Brasil, laisa@ufrj.br, https://orcid.org/0000-0002-4573-0969

Palabras-clave: Formación de profesores. Análisis crítico del discurso. Lucha contra hegemónica. Educación ambiental. Prácticas universitarias.

\author{
Keywords: \\ Teacher training. \\ Critical analyze of \\ discourse. Resistance. \\ Environmental \\ education. University \\ practices.
}

RESUMEN: Este artículo presenta un análisis de eventos comunicativos de esperanza en la lucha contra hegemónica desde prácticas de educación ambiental al interior de una universidad y en un programa de licenciatura en Colombia. Dichos eventos comunicativos fueron identificados a partir del análisis crítico del discurso de una entrevista realizada a dos profesores del componente socioambiental de una licenciatura en ciencias naturales y educación ambiental en Colombia. En relación con el escenario universitario, establecimos algunas categorías generales para la comprensión de prácticas ambientales de resistencia que pudieran aparecer en las selecciones discursivas de los profesores entrevistados, entre ellas contenido, relación, reacción, acción y contenido expositivo. Respecto al escenario de la licenciatura, se consideró acciones educativas hipotéticas de deseo y prácticas educativas alternativas que buscaban la superación de la coerción ejercida por la estructura curricular. En los resultados, encontramos la existencia de algunas prácticas ambientales bajo la ideología de democracia y justicia socioambiental; adicionalmente términos como "oposición"; "pelean", "no aceptan", "muy tocados", y "se volcán a prestar atención" fueron claves para considerarlas como parte del movimiento de resistencia política-cultural. Algunas prácticas universitarias hacían referencia a la política ambiental, el movimiento estudiantil y la feria ambiental; con relación a la licenciatura aparece como fundamental la formación política para fomentar a su vez una movilización civil, también la necesidad de crear prácticas de resistencia a la industria como por ejemplo la producción alimenticia a partir de biotorres. Con base a los resultados, argumentamos que diferentes escenarios existentes en el contexto universitario, pueden ser claves en los procesos de formación ya que permiten al estudiante tener una agencia, contribuyendo a una formación basada en cooperación, actividad política, crítica y participación ciudadana.

ABSTRACT: This paper presents some communicative events that show hope through the resistance of hegemonic from environmental education practices inside a university and in a bachelor degree in Colombia. To collected data were applied an interview with two professors of the socio-environmental component of a degree in natural sciences and environmental education in Colombia, and to analyzed it with used our theoretical and methodological framework of critical analyzes of discourses. About the university scenario, we established some general categories for the understanding of environmental resistance practices that could appear in the discursive selections of the professors interviewed, including content, relationship, 
reaction, action, and exhibition content. We considered for the undergraduate scenario, the hypothetical educational actions, and alternative educational practices that sought to overcome the coercion exerted by the curricular structure. In the results, we found the existence of some environmental practices under the ideology of democracy and socio-environmental justice; additionally, terms such as opposition, they fight, they don't accept, very touched and they pay attention were key to consider them as part of the political-cultural resistance movement. Some university practices referred to environmental policy, the student movement and the environmental fair; Concerning the degree, political training is essential to promote civil mobilization, as well as the need to create practices of resistance to the industry, such as food production from bio towers. Based on the results, we argue that different scenarios existing in the university context can be key in the training processes since they allow the student to have an agency, contributing to a training based on cooperation, political activity, criticism and citizen participation.

\section{INTRODUCCIÓN}

La formación de profesores es basada por diferentes campos de conocimiento, a su vez por diferentes comunidades epistémicas que pueden ser excluyentes entre sí, dado por sus concepciones de educación, ciencia, investigación y/o ideologías. En este mismo escenario, el estatus de la ciencia puede variar de una comunidad a otra, en algunos casos pueden asumir que esta es de carácter neutro o en otros cargada de ideologías, valores como toda producción social. Traemos estos elementos a debate porque dentro de la formación de profesores podemos encontrar por tanto posiciones hegemónicas del componente científico, y posiciones subordinadas en las prácticas educativas, como podría ser el caso de la educación ambiental.

En este contexto, en una licenciatura que posee tanto la formación científica y la educación ambiental, es necesario pensar, debatir y crear estrategias de generar distanciamiento de los modelos totalizadores de la ciencia tradicional estableciendo encuentros con otras áreas de conocimiento (JAVIER; CASTRO ROSALES, 2016). Por consiguiente, traemos a consideración una educación ambiental, que, al presentar las especificidades de la discusión ambiental, tiene como objetivo discutir los discursos hegemónicos y los modelos de sociedad, desarrollar una formación política, así como reconocer prácticas materiales de alienación simbólica (ANDRADE RODRIGUES; DE LUCA; SORRENTINO, 2012; ARDOIN; CLARK; KELSEY, 2012; BONNETT, 2013; CARVALHO; TOMAZELLO; OLIVEIRA，2009; GRUENEWALD，2004; JICKLING; WALS, 2008; LOUREIRO, 2006; LOUREIRO; GIRÃO; LIMA, 2009; MARCOS, 2012; SORRENTINO et al., 2005; TORRES M; BARRIOS E, 2009). Acompañada de una perspectiva epistemológica de realismo crítico, que en palabras de Lotz-Sisitka y Price (2016) "soporta la ciencia social objetiva a través de la investigación empírica de poderes causales o mecanismos generativos que existen en diferentes niveles de la realidad, ya sea que los conozcamos, reconozcamos o hagamos referencia a ellos o no". Esto significa que, en un contexto dominado por algunas fuerzas, buscamos puntos de ruptura o posibles caminos de 
emancipación. Desde la lectura de contexto, buscamos iluminar proporcionando así recursos que se pueden utilizar para una formación de profesores más integral cargada de insurgencias, esperanzas y resistencias, al reconocer que la práctica educativa no es totalmente libre. En ese sentido, son los mecanismos que forman los eventos y por tanto los acontecimientos, por consiguiente, se debe considerar los mecanismos imprevistos tanto estructurales como agénciales, dado que no se puede realizar un análisis causa-efecto lineal (BHASKAR; COLLIER, 1998; PARRA HEREDIA, 2016).

Dentro de algunas premisas del realismo crítico, asumimos que la estructura social es condición necesaria para cualquier acto intencional y su pre-existencia establece su autonomía como posibles objetos de investigación científica y que su poder causal establece su realidad (BHASKAR, 1998). Por consiguiente, nuestra investigación reconoce la estructura social, sus dinámicas en la producción de conocimiento y sus relaciones de poder para establecer la crítica explicativa en los procesos educativos en el que están los temas ambientales. Esto significa que la formación de profesores pertenece a una estructura social, en nuestro caso la colombiana con influencias internacionales, con sus dinámicas propias que responden a criterios institucionalizados, lineamientos políticos, de tal forma que influencia los procesos investigativos de esta (MEJÍA-CÁCERES, 2019). Por otro lado, el modelo de transformación de la actividad social desarrollado aquí implica una concepción relacional del objeto de la ciencia social. En este sentido, hemos creado un modelo relacional en el cual ubicamos la formación de profesores de Colombia, en relación con otras dimensiones como la política, el currículo, el proceso de aprendizaje y construcción socio cognitiva, el discurso; investigando los micro y macroprocesos sociales ${ }^{1}$.

Así a través de la figura 1, expresamos una sociedad donde el conocimiento tiene estatus de poder generando, por ejemplo, que la política restrinja la autonomía de los procesos educativos, sin embargo, esa política es interpretada y traducida organizando propuestas de currículo, que a su vez constituye la práctica de la formación de profesores (BALL; MAGUIRE; BRAUN, 2012; MAGUIRE et al., 2011). Esta contribuye a construir a nivel individual y colectivo conocimientos, lenguajes y experiencias, que interactúan entre sí, y que se materializan a través del discurso. En el discurso los sujetos interactúan, participan, interpretan, se oponen o apoyan a los procesos micro y macro sociales. Al mismo tiempo que proponen elementos del dominio de lo realizado, eventos o actividades que son realizadas generan efectos de poder, que pueden ser explícitos o no (BARROS, 2015). En este sentido, el análisis contribuye a entender dichos procesos y a proponer puntos de quiebre en la

\footnotetext{
${ }^{1}$ Entendemos como procesos macro sociales aquellos de gran escala, como el estado, la cultura, las clases sociales, y micro social, experiencias y actores sociales, en este último se puede hacer un análisis detallada de sus discursos (COLLINS, 1981).
} 
relación hegemónica de la política y del conocimiento científico, descripto anteriormente, de ahí que la elipse tiene línea discontinua, haciendo referencia a la posibilidad de transformación social.

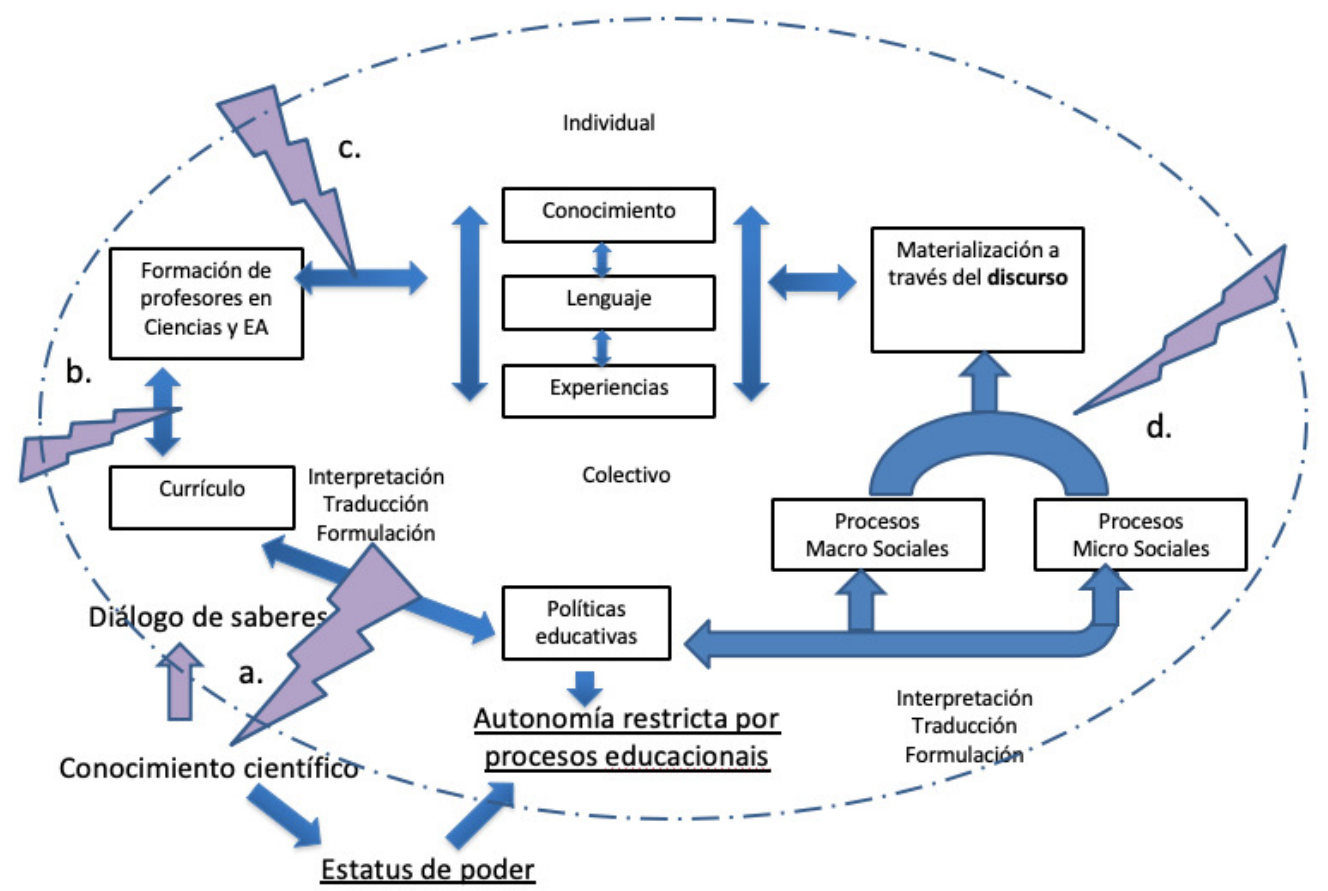

Figura 1 - Modelo relacional inicial de los componentes de la investigación. Representamos la estructura social a través de una elipse, en la cual está inmersa la formación de profesores en Colombia. La figura está compuesta por: 1. rectángulos, en los cuales tan inmersas las dimensiones: política; currículo; proceso de aprendizaje y construcción socio cognitiva, discurso y procesos micro y macro sociales. 2. Las flechas, pueden ser de doble sentido, indicando la existencia de una mutua influencia entre las dimensiones, en algunas ocasiones es usada la flecha de único sentido, una indicando influencia directa tanto a nivel hegemónico como de ruptura. 3. Los rayos, significan puntos de ruptura o quiebre. 4. Los colores, el azul indicando relaciones "naturalizadas" y el purpura indicando relaciones de ruptura.

Fuente: Mejía-Cáceres, 2019.

Lo que estamos queriendo decir con eventos comunicativos son las interacciones sociales que se desarrollan en un marco espacio-temporal especifico, de ahí que los participantes intercambian algún tipo de información, sentimiento y/o sensación (RAFFO, 2002; RAITER, 2003). Para Van Dijk (2015, 2016), la estructura social tiene control sobre los eventos comunicativos a través de los grupos de poder, instituciones y élites simbólicas; dentro de los eventos comunicativos, encontramos la situación de comunicación en relación con la estructura discursiva, en la cual la interacción social se da a través del escenario (tiempo, lugar), participantes, identidad, roles, relaciones, propósitos, conocimiento, ideologías, acción social, actos de habla, entre otros. Esto influye en los modelos de contexto y situación personal, en otras palabras, ideologías, actitudes sociales y conocimiento sociocultural, como expresamos a través de la figura 2. 


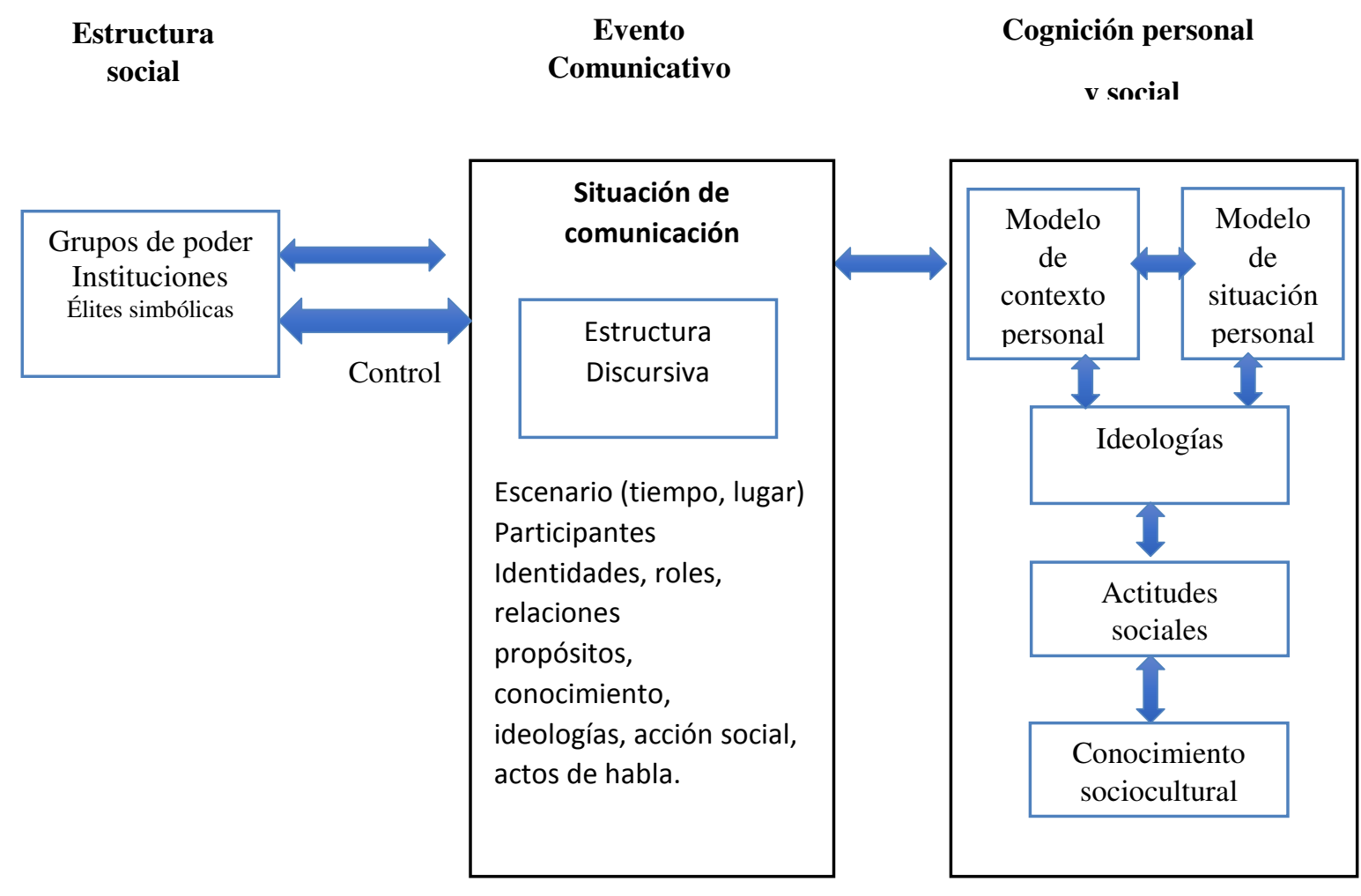

Figura 2: Esquema de la reproducción discursiva del poder. Fuente: Adaptado de VAN DIJK, 2015, 2016.

Considerando lo anterior, este artículo se ha estructurado de la siguiente forma: primero, una descripción del contexto investigativo de una licenciatura en Colombia que presenta una singularidad en la formación de profesores de ciencias al considerar la Educación Ambiental como ámbito de la formación a nivel de pregrado en los objetivos, proyecto pedagógico y titulación del docente. Posteriormente, tratamos de entender y caracterizar las prácticas ambientales universitarias que pueden aportar a la formación política del licenciando, seguido de las prácticas de educación ambiental en la licenciatura. Debatimos que, la entrevista como evento comunicativo y analizada a partir de los estudios críticos del discurso, ha posibilitado la crítica para entender las posibilidades de esperanza en el contexto de Educación Ambiental en la licenciatura.

Dos profesores del componente socio-ambiental respondieron positivamente a la participación en la entrevista, Marcela y Paulo (nombres ficticios). Marcela posee maestría y especialización en Enseñanza de las ciencias y tiene 16 años de experiencia en la universidad, es profesora nombrada. El profesor Paulo es contratista y está hace 4 años en la universidad, es especialista y tiene una maestría en Educación Ambiental. La formación a nivel de pregrado de los dos es en el área de biología y química. 
En cuanto a nuestro método de generación de datos, fue diseñada una entrevista semiestructurada $^{2}$ la cual fue validada por cinco expertos de diferentes universidades. Fue estructurada en cuatro categorías, la primera de ellas referente al contexto de influencia ${ }^{3}$, con dos preguntas; la segunda sobre posicionamientos y acciones políticas, con tres preguntas, la tercera sobre las prácticas educativas (dos preguntas) y la cuarta sobre la sistematización de estas a través de las monografías de la licenciatura. Correspondiente al procesamiento de datos, una vez realizadas las entrevistas procedimos a realizar transcripción. Para el análisis de las entrevistas, tuvimos en cuenta las estrategias discursivas usadas en cada uno de los textos transcriptos, realizando primeramente una lectura general del documento, y un análisis textual, para proceder a realizar un análisis articulado al contexto. "La semántica del discurso debería tener en cuenta no sólo el significado de las estructuras más allá del límite de la frase, sino también las posibilidades de dependencia del discurso -significado de palabras, frases, cláusulas y oraciones y sus relaciones mutuas" (VAN DIJK, 2008, p. 220). En este sentido, caracterizamos aspectos de la dimensión textual como la modalidad, los actos de habla, presuposiciones, intertextualidad; permitiendo la comprensión de la dimensión cognitiva y de la dimensión social.

\section{LA EDUCACIÓN AMBIENTAL EN LA LICENCIATURA EN CIENCIAS NATURALES Y EDUCACIÓN AMBIENTAL EN COLOMBIA}

En esta investigación, trabajamos con la Licenciatura con Énfasis en Ciencias Naturales y Educación Ambiental de la Universidad del Valle. Cuenta con acreditación de alta calidad por el Ministerio de Educación Nacional de Colombia (MEN). Como objetivo general se presenta formar Licenciados en Educación Básica con Énfasis en Ciencias Naturales y Educación Ambiental, con competencias académicas, investigativas y humanas que le permitan desempeñarse exitosamente en las instituciones educativas de $1^{\circ}$ a $11^{\circ}$ grado. La licenciatura hasta el año 2016 estuvo organizada por cinco elementos: las asignaturas básicas, asignaturas profesionales, electivas complementarias, electivas profesionales y asignaturas obligatorias de ley. Las asignaturas básicas y profesionales estaban agrupadas en cuatro componentes: el conocimiento socio ambiental, conocimiento científico, conocimiento pedagógico y conocimiento didáctico. En el eje socioambiental los estudiantes contaban con las asignaturas obligatorias: Historia y Educación Ambiental, Cultura del paisaje, Problemas

\footnotetext{
2 Para mayor información revisar Mejía-Cáceres, 2019.

${ }^{3}$ Ball \& Bowe (1992), proponen el ciclo de política (parte de nuestro referencial teórico-metodológico) la cual está conformada por tres tipos de contexto. El primero de ellos es el contexto de influencia, en el cual las políticas y discursos son iniciados y construidos; el segundo es el contexto de la producción de texto materializado en textos políticos legales; el tercero es el contexto de la práctica, escenario donde la política está sujeta a la interpretación y recreación, donde produce efectos y consecuencias, cambios y transformaciones.
} 
Ensino, Saúde e Ambiente - Número Especial, pp. 239-254, Junho 2020

ambientales I y II, Educación Ambiental y Desarrollo Sostenible, Proyectos ambientales escolares. Confiriendo un espacio curricular privilegiado a la Educación Ambiental. Con la reestructuración curricular del año 2017 los componentes son: fundamentos generales, saberes específicos y disciplinares, pedagogía y ciencias de la educación, didáctica de las disciplinas y electivas profesionales, restringiendo el campo de acción de la Educación Ambiental.

Mejía-Cáceres (2019), al analizar los objetivos y la nueva estructura curricular, identifica la Educación Ambiental como objetivo de formación independiente de la Educación en Ciencias, sin embargo, al analizar la matriz curricular observa como la Educación Ambiental está incluida en una licenciatura en ciencias y que los cambios sufridos reducen los aportes de Educación Ambiental a la formación de los estudiantes y además, la mayoría de las asignaturas del componente de didáctica de las disciplinas están direccionadas para la Educación en Ciencias a pesar de que la Educación Ambiental permanece en los objetivos del programa. De esta manera, se reproduce la hegemonía del discurso dominante de la ciencia sobre el currículo, y perpetua la relación de inclusión de Educación Ambiental -Educación en Ciencias y no una relación de asociación (MEJÍA-CÁCERES, 2019). Adicionalmente, no se encuentra explícito dentro de los objetivos y la estructura curricular, elementos sobre la formación para actuación política del profesor y espacios de formación socio ambientales en amplia perspectiva. Por consiguiente, indagamos, ¿a qué instancias de la formación del profesor los eventos comunicativos se refieren como posibilidades de ruptura de hegemonías? Para debatir la cuestión, recurrimos a Lopes \& Macedo (2011, p. 19) que consideran el currículo como una esfera amplia que involucra diferentes instancias de aprendizaje. En sus palabras, se entiende el currículo como "la organización previa o no de experiencias/situaciones de aprendizaje realizada por profesores/redes de enseñanza de forma a llevar a cabo un proceso educativo" Por ende, concordamos con Moreira e Silva (1995) al definir el currículo como un artefacto social y cultural condicionado por la estructura social en lo cual se construye, en donde se materializan relaciones de disputa de poder por conocimientos a enseñar.

\section{EL AMBIENTAL EN LAS PRÁCTICAS UNIVERSITARIAS: APORTES A LA FORMACIÓN POLÍTICA DEL LICENCIANDO A TRAVÉS DEL MOVIMIENTO ESTUDIANTIL}

En este sentido, las prácticas descritas aquí son encontradas en la vida académica en general y en las acciones socio ambientales al interior de la universidad. Asimismo, en los discursos de los profesores entrevistados encontramos términos con relación a las prácticas 
ambientales realizadas dentro de la Universidad del Valle, que se pueden identificar bajo una ideología democrática y de justicia socio ambiental. De ahí, que, de acuerdo con la selección discursiva de la profesora Marcela, quien presenta positivamente algunas prácticas estudiantiles, procedimos a identificarlas y organizarlas de acuerdo con el escenario universitario, a categorías generales (contenido, relación, reacción, acción y contenido expositivo). Las reacciones y acciones descritas en el escenario universitario en el ámbito del movimiento estudiantil fueron de oposición, movilización, defensa de la fauna del campus y lucha por espacios ambientales. Para identificar estas prácticas nos basamos en los

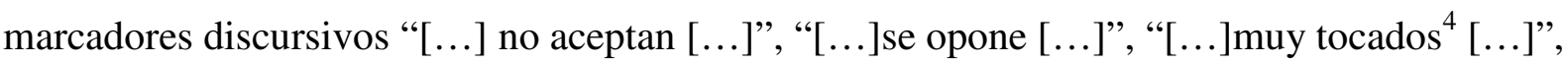
“[...]se volcán a prestar atención [...]”, “[...] pelean [...]”, de esta manera, la profesora Marcela introduce en sus discursos algunas marcas de resistencia, es decir, formas de protestar contra un poder opresor o luchar contra cualquier tipo de dominación.

En el fragmento abajo (negritas indican los actores y acciones de ellos), la profesora Marcela presenta a los estudiantes como actores sensibles, activos, reactivos y que pelean por la cuestión ambiental en el campus de la universidad. Al ser indagada ¿Cómo la vida universitaria genera espacios de participación o de creación acciones de lucha y resistencia ante una injusticia ambiental ${ }^{5}$ Marcela hace referencia a los estudiantes en general y al nombrar también la población, amplia la discusión (no solamente se refiere a los estudiantes de la universidad) presentando una dimensión de lucha y resistencia socio ambiental del país y puede ser que el "aquí” sea en Colombia:

Pues, cuando nosotros presentamos todas estas problemáticas los estudiantes en sus discusiones críticas no aceptan el trato que se da, por ejemplo, a nivel de Colombia con el nuevo presidente que tenemos en una de sus campañas, él por ejemplo estaba apoyando la práctica de extracción de petróleo con agua, que era el fracking, y los estudiantes nuestros son supremamente sensibles frente a eso, entonces la población se opone a ese tipo de prácticas aquí [...] (E-M, líneas 1388 a la 1392).

[...] ellos han creado una ciclo ruta, pero resulta que la universidad ha aumentado el número de vehículos al interior del campus, entonces no se están respetando las rutas que los muchachos hicieron para que circularan las bicicletas... pelean porque los carros están obstaculizando, taponando las vías que ellos ya hicieron con demarcaciones y todo [...] (E-M, líneas 1400 a la 1406).

Identificamos la movilización estudiantil a partir de prácticas de oposición, movilización, defensa y lucha por situaciones de vulnerabilidad ambiental generada dentro de la base sociopolítica colombiana referente a la dimensión ambiental, a partir de nuevas prácticas como el fracking o de reestructuración del plan ordenamiento territorial implicando

\footnotetext{
${ }^{4}$ Esta expresión es un modismo propio de Colombia que hace referencia a un compromiso frente a una determinada situación.

${ }^{5}$ El profesor Paulo trató de dichas cuestiones por un aspecto de ciudadanía.
} 
modificar el ecosistema en algunas zonas. De ahí, que los estudiantes tienen una tendencia de defensa y lucha al interior del campus universitario, en la búsqueda de espacios que permitan una mejor calidad de vida tanto para los actores sociales que frecuentan el campus, como de los animales que viven en este.

Considerando a Agbedahin \& Lotz-Sisitka (2019) cuando debate que "las acciones o prácticas previas, inmediatas o futuristas de los agentes humanos en y alrededor de las estructuras sociales, determinan si la estructura social denotada por posiciones será reproducido o transformado", en este sentido, la influencia de la posición de profesores encargados de la formación inicial de profesores es substancial y determinante, de ahí, que si se generan movimientos de resistencia política-cultural se estará contribuyendo a la lucha contra hegemónica de la Educación Ambiental (MEJÍA- CÁCERES et al., 2017). Precisamente, Marcela trae luchas socio ambientales al evento comunicativo indicando su compromiso con ellas. Además, Marcela presenta un posicionamiento crítico frente a la política ambiental de la universidad. La misma recibe una valoración negativa por la profesora, aunque ella este representándose a sí misma como un enunciador de un grupo. Dentro de las críticas que realiza a la política ambiental de la universidad, es el enfoque naturalista, ya que desde este tipo de enfoques se desconoce el componente social, a su vez, hace referencia al "desconocimiento" o "negligencia" de articular con la Política Nacional de Educación Ambiental de Colombia. Sin embargo, retomamos desde nuestro referencial teórico del ciclo de políticas ${ }^{6}$, que esta tiene un proceso de interpretación y traducción, por tanto, asumimos que la política ambiental es producto de la interpretación y traducción de la Política Nacional de Educación Ambiental por un grupo de actores sociales de la Universidad.

Adicionalmente, aparece la feria ambiental como una práctica universitaria liderada por la facultad de ingeniería ${ }^{7}$. Sin embargo, esta práctica recibe una evaluación negativa por parte de la profesora Marcela al denominarla naturalista, al igual que la política ambiental. A su vez, introduce la existencia de incoherencia entre lo "hablado" y lo "realizado" por "ellos", dado que se asumen desde una perspectiva sociocultural, lo que implicaría discusiones más amplias, pero a nivel de lo concreto, quedan en una visión pragmática y vinculada a la gestión de espacio.

En suma, consideramos importante resaltar los diferentes escenarios que la universidad permiten a los estudiantes tener una agencia, de esta manera, se genera una formación

\footnotetext{
${ }^{6}$ Ver nota 2

${ }^{7}$ Aclaramos que las actividades fueron identificadas por la profesora Marcela a través de un proyecto de investigación en compañía con el área de ingeniería, a su vez, legitima el vínculo con otras áreas de conocimiento y profesores de otras facultades, ya que permite realizar proyectos solicitados por el Ministerio de Ambiente.
} 
orientada hacia la cooperación, la actividad política, la crítica y la participación ciudadana aunque con diputas y conflictos internos. Si por un lado, observamos que hay espacios de resistencia, a la vez, los retos se sitúan en reconocer que el enfrentamiento de las cuestiones ambientales locales presentadas en las entrevistas es resultado de un cientificismo discursivo como plantea Bonnet (2013). Para él, el paradigma de ciencia aplicada, ha sido y fue la base de la Educación Ambiental desde los años de 1980, generando por su vez, un instrumentalismo y antropocentrismo que constituyó un marco cultural y que entendemos haber adentrado los espacios educativos.

\section{PRÁCTICAS DE EDUCACIÓN AMBIENTAL EN LA LICENCIATURA}

Traer la esperanza en la actualidad, donde las cuestiones educativas y socio ambientales son a cada día atacadas por grupos que defienden el avanzo del neoliberalismo y ultraderecha, es entenderla como parte de las utopías de la llegada. Significa que, es vislumbrar la esperanza cargada de acciones y prácticas que buscan la superación de modos de ser y accionar que ya están naturalizados en los macro procesos sociales y constitutivamente en los micro procesos como el caso de nuestro objeto de estudio que son las propuestas curriculares de EA en la licenciatura. Así, el romper con visiones hegemónicas y trabajar buscando el fortalecimiento de la colectividad, la identidad cultural y las prácticas alternativas es parte del argumento que hemos construido. Preguntarnos ¿Cómo la licenciatura a partir de la Educación Ambiental participa o crea acciones de lucha y resistencia ante una injusticia ambiental? Nos lleva a las selecciones discursivas realizadas por el profesor Paulo, quién cuestiona la ausencia de reconocimiento de patrones de marcación y alienación de la carga ideológica mediante la formación y considera que para crear acciones de resistencia se debe iniciar con ejercicios micro etnográficos y micro semióticos, seguido de una formación de sujetos con actitudes eco-críticas con relación a la influencia ideológica, conllevando a la búsqueda de la liberación de paradigmas educativos que encasillan los procesos de educación y que restringen la libertad de cátedra a través de la estructura curricular, lo cual nos recuerda a Gil (2017) quien afirma que, "el concepto de resistencia pedagógica se caracteriza por estar ligada a formas de conocimiento y de comprensión que permiten generar creativamente políticas educativas nacidas de las luchas, de las experiencias". En este sentido, el reconocimiento de prácticas de resistencia que se dan al interior de la licenciatura y de la universidad que en la mayoría de los casos son naturalizadas o excluidas en discusiones y debates dentro la formación de profesores, pueden contribuir a generar nuevos caminos de reflexión y transformación a partir de la política educativa propia de la licenciatura. 
Algunas reacciones y acciones descritas en el caso del escenario de la licenciatura son de hipotético de deseo, es decir, se deben crear o potenciar, este es el movimiento civil, el cual está fuertemente articulado con el escenario universitario en el cual identificamos el movimiento estudiantil. Sin embargo, en este caso, la intención del profesor Paulo es ampliar el escenario del "profesor en formación" por su escenario de "ciudadano", trayendo consigo no solamente la relación de resistencia sino de responsabilidad civil. En este sentido, el profesor Paulo articula una formación no solamente ecológica sino política, contribuyendo así a un pensamiento crítico, como se muestra en el siguiente fragmento (negritas indican los actores, opresión de estos y posibles acciones de ellos), lo que requiere "de una posición de unos discursos y prácticas curriculares democráticas, que evitan posturas de sumisión, como de indiferencia y de silencio cómplice"(GIL CLAROS, 2017).

[...] también puede contemplarse aspectos de opresión vivida, por ejemplo, por los sujetos, y ser muy crítico con los mismos programas universitarios de educación que tenemos o de investigación o de extensión que hay en a la universidad, por ejemplo, que tipo de acciones de lucha, resistencia o de injusticia se generan por ejemplo en las mismas universidades con los estudiantes [...] (E-P, 1748 a la 1752).

En este sentido, respecto a nuestra pregunta encontramos que las acciones de lucha y resistencia inician con el reconocimiento de la opresión vivida por ellos mismos como estudiantes, al igual que a través del reconocimiento del límite de esa realidad opresora (FREIRE, 2016). Lo que indica, que el profesor Paulo hace lectura que la formación del licenciando y la universidad están inmersas en relaciones de poder que responden a una pedagogía de la estandarización y de competencias creadas por el sistema neoliberal. Para Mejía-Cáceres (2019) aunque la misma universidad impone opresión sobre los estudiantes estos pueden tener acciones liberadoras. Esto implica considerar, que los estudiantes deben recibir una formación política para tener la capacidad de "desarrollar su propio juicio político, tomar sus propias decisiones y desarrollar su acción responsable dentro de la sociedad democrática”(LOBATÓN-PATIÑO, 2016, p. 259).

Para Lobatón -Patiño (2016), la formación política se da mediante las competencias del juicio político, las competencias de la acción políticas, las competencias metódicas y las competencias relacionadas con el saber de la especialidad. En este sentido, el profesor Paulo hace referencias al juicio político, que implica analizar el contenido, forma, proceso y efectos de las decisiones políticas, para poder realizar la acción política, al afirmar que la educación debe transformar el bio-sujeto para actuar como una acción política pero al servicio del paradigma amo-colonial-foráneo; en otras palabras, el profesor Paulo reconoce la capacidad de agencia del estudiante como ciudadano, una agencia relativa, de poderes causales, y una realidad hegemónica que estaría condicionando esta agencia. 
Identificamos el profesor Paulo, como un profesor crítico que intenta transformar su práctica educativa para no mantener y reproducir las hegemonías propias de la práctica educativa, que para él están basadas en epistemologías de sometimiento. En este sentido, encontramos en el siguiente fragmento, una práctica educativa que aporta a la formación disciplinar, pero también a una formación política, veamos:

[...] ejemplo tenemos la construcción de bio terrazas enteras, o mejor las biotorreras que tenemos ahora, son donde ellos por ejemplo aprenden a integrar las ciencias multidisciplinaria y comienza en estas bio torres comienzan a sembrar por ejemplo especies de importancia para la canasta familiar, y ellos ahí van cuidando la semilla, van aprendiendo de las distintas ciencias [...] y para ellos es una obra y entonces eso es como, digamos un referente para confrontar el, digamos la industria alimenticia [...] (E-P, 1819 a la 1828).

Si analizamos el fragmento anterior de acuerdo con la formación política, asumimos que el profesor Paulo está desarrollando no solamente la competencia de juicio sino de acción política, ya que está formando para entrar en controversias políticas conflictivas y llegar a compromisos, reflexionar acerca de comportamiento de consumidor en relación con decisiones económicas y ambientales (LOBATÓN-PATIÑO, 2016). Permitiendo en términos de Lobatón-Patiño una articulación entre argumentos, comportamientos y decisiones, al usar el conocimiento científico para comprender, cuestionar y argumentar ciertas prácticas sociales (como es el caso de la agricultura a nivel masivo), generar cambios en los comportamientos al buscar prácticas alternativas (la biotorre), y decisiones que pueden ir a diversas dimensiones como sujeto ciudadano.

Por consiguiente, este tipo de prácticas educativas permiten tomar distanciamiento de los modelos totalizadores de la ciencia tradicional, de ahí que evidenciamos que existen espacios en el currículo que pueden superar hegemonías internas de la licenciatura como es aquella ligada al conocimiento científico como poder, al permitir diálogo con otras áreas de conocimiento como es el caso de la sociología, la filosofía, al permitirnos debatir sobre los modelos de la sociedad y desarrollar una formación política.

Por tanto, identificamos que los enfoques, temáticas y actividades didácticas son diferentes de acuerdo con la cognición social del profesor, de esta manera, no se puede afirmar si la licenciatura realiza fuertemente o no, acciones de reproducción o resistencia a procesos hegemónicos. De ahí, que reafirmamos la posibilidad de la existencia o creación de puntos de ruptura en la práctica educativa como por ejemplo, asumir una perspectiva sociocultural, realizar prácticas y actividades de denuncia, participar, debatir, analizar los movimientos estudiantiles y civiles, y sus aportes a la formación del licenciando, la creación, fortalecimiento de prácticas alternativas que permitan no solamente una comprensión y argumentación disciplinar sino que contribuyan a la resistencia y oposición del modelo 
económico. Lo cual podrá influenciar micro procesos sociales a partir del fortalecimiento de la colectividad, el fortalecimiento de la identidad cultural, la generación de prácticas alternativas, como resultado de una formación integral y política.

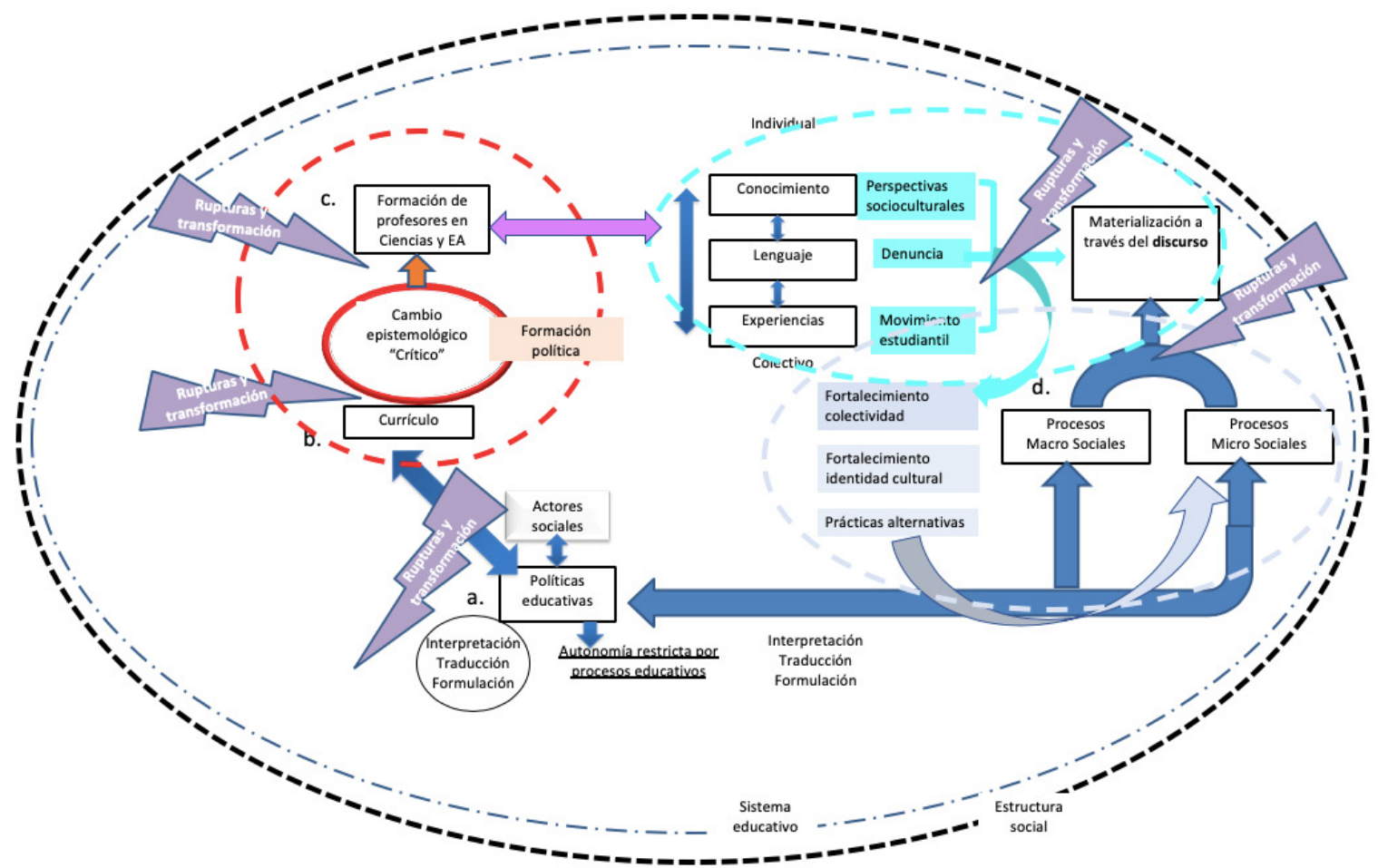

Figura 3: Modelo relacional de rupturas de la práctica de EA. Esta figura hace énfasis en las posibles rupturas dada desde la cognición social a través de la perspectiva sociocultural, lenguaje mediante la denuncia y experiencia, como por ejemplo, los movimientos estudiantiles, articulados a los procesos de formación de profesores, representados por el color agua marina, los cuales a su vez contribuyen a modificar microprocesos sociales, al fortalecer la colectividad, la identidad cultural, y el diseño de prácticas alternativas al sistema, como por ejemplo, las biotorres, que favorecen una seguridad alimentaria, representado por el azul claro.

Fuente: Adaptado de MEJÍA-CÁCERES, 2019.

Por tanto, a través del análisis de los eventos comunicativos encontramos posibles caminos de emancipación, posibles puntos de ruptura que dan esperanza, y permiten contribuir a visualizar nuevos caminos para la formación inicial de profesores aún cuando esta es condicionada por el sistema.

\section{CONSIDERACIONES FINALES}

Este estudio permitió argumentar como prácticas al interior de la universidad que han sido naturalizadas o simplemente ignoradas, pueden contribuir a una formación crítica y política para los licenciados, sobre todo considerando que la educación es un medio que debe contribuir a la transformación social. También argumentamos como eventos comunicativos están cargados de poder, de ahí que es posible considerar la posibilidad de cambio en diferentes niveles. Los resultados encontrados de las prácticas de ruptura demandan al proceso de formación de profesores más que un currículo centrado en la disciplina, sino que también 
es importante dar atención a los eventos tanto en la licenciatura como en la universidad en general que contribuyen al fortalecimiento da agencia de sujetos como ciudadanos activos en la transformación social. En este sentido, reflexionar respecto la universidad como un locus pedagógico para los profesores de formación amplía la posibilidad de resistencia a las estructuras opresoras y a la esperanza de procesos educativos que actúen en posibilidades de campo y creación de nuevas utopías.

Valorizar los movimientos estudiantiles como escenarios de empoderamiento estudiantil y ciudadano en la búsqueda de la justicia socio-ambiental, lo que a su vez esta articulado con el diálogo de saberes, con diferentes áreas de conocimiento y experiencias que buscan alternativas al status quo parece ser un camino de esperanza y que compone el currículo de profesores en formación

\section{AGRADECIMIENTO}

La presente investigación fue realizada con el suporte financiero de Coordenação de Aperfeiçoamento de Pessoal de Nível Superior - Brasil (CAPES) - Código de Financiamiento 001.

La presente investigación fue realizada con el suporte financiero de CNPq.

\section{REFERENCIAS BIBLIOGRÁFICAS}

AGBEDAHIN, A.; LOTZ-SISITKA, H. Mainstreaming education for sustainable development: elaborating the role of position-practice systems using seven laminations of scale. Journal of Critical Realism, 2019.

ANDRADE RODRIGUES, D.; DE LUCA, A.; SORRENTINO, M. O diálogo em processos de políticas públicas de educação ambiental no Brasil. Educação e Sociedade, v. 33, n. 119, p. 613-630, 2012.

ARDOIN, N. M.; CLARK, C.; KELSEY, E. An Exploration of Future Trends in Environmental Education Research. Environmental Education Research, n. November, p. $1-22,2012$.

BALL, S.J., BOWE, R. Subject departments and the implementation of National Curriculum policy: an overview of the issues. Journal of Curriculum Studies, v. 24, p. 97-115, 1992.

BAll, S.; MAguiRe, M.; BRAUn, A. How Shools do Policy: Policy Enactments in Secondary Schools. New York: Routledge, 2012.

BARros, S. M. Realismo Crítico e Emancipaçao Humana. Campinas, SP: Pontes Editores, 2015.

BHASKAR, R. Societie. In: ARCHER, M. et al. (Eds.). . Realism Critical: Essential Readings. London: Routledge, 1998. p. 206-257.

BHASKAR, R.; COLLIER, A. The theory of explanatory critiques. In: Critical Realism: Essential Readings. [s.1.] Routledge, 1998.

BONNETT, M. Normalizing catastrophe: sustainability and scientism. Environmental 
Education Research, v. 19, n. 2, p. 187-197, 2013.

CARVAlHO, L. M. DE; TOMAZELlo, M. G. C.; OliveIRA, H. T. DE. Pesquisa em Educação Ambiental: Panorama da Produção Brasileira e Alguns de seus Dilemas. Cadernos Cedes, v. 29, n. 77, p. 13-27, 2009.

COLLINS, R. The Microfoundations of Macrosociology. In: Sociology Since Midcentury. Essays in Theory Cumulation. New York: Academic Press Inc, 1981.

FREIRE, P. Pedagogia do oprimido. 62 ed. ed. Rio de Janeiro: Paz \& Terra, 2016.

GIL CLAROS, M. G. Educación, subjetividades y pensamiento crítico. Boletin Virtual, v. 67, n. Julio, 2017.

GRUENEWALD, D. A. A Foucauldian Analysis of Environmental Education: Toward the Socioecological Challenge of the Earth Charter *. Curriculum Inquiry, v. 34, n. 71-107, 2004.

JAVIER, R. R.; CASTRO ROSALES, E. La Educación Ambiental: ¿Un Campo de Conocimiento? Revista Electrônica do Mestrado em Educação Ambiental, v. 33, n. 1, p. 95-11, 2016.

JICKLING, B.; WALS, A. E. J. Globalization and Environmental Education: Looking Beyond Sustainable Development. Jornal of Curriculum Studies, v. 40, n. 1, p. 1-21, 2008.

LOBATÓN-PATIÑO, R. El ámbito científico de la formación política: elementos conceptuales y líneas de investigación. Educación y Educadores, v. 19, n. 2, p. 254-273, 2016.

LOPES, A. C.; MACEDO, E. Teorias de currículo. São Paulo: Cortez Editora, 2011.

LOTZ-SISITKA, H.; PRICE, L. Critical Realism, Environmental Learning and Sociolecological Change. Oxon: Routledge, 2016.

LOUREIRO, C. F. Complexidade e Dialética: Contribuições à práxis Política e Emancipatória em Educação Ambiental. Educação e Sociedade, v. 27, n. 94, p. 131-152, 2006.

LOUREIRO, C. F.; GIRÃO, J.; LIMA, S. DE. Educação ambiental e educação científica na perspectiva Ciência, Tecnologia e Sociedade ( CTS ): pilares para uma educação crítica. Acta Scientiae, v. 11, n. 1, p. 88-100, 2009.

MAGUIRE, M. et al. Policy discourses in school texts. Discourse: Studies in the Culturas Politics of Education, v. 32, n. 4, p. 597-609, 2011.

MARCOS, R. J. La política Nacional de Educación Ambiental a la Luz del Cielo de las Políticas Públicas. Hontanar, v. 14, n. 1, p. 71-88, 2012.

MEJÍA- CÁCERES, M. A. et al. Perspectivas críticas de educación ambiental: Abordando cuestiones de vulnerabilidad socioambiental en la eneseñanza de las ciencias. In: Multiculturalidad y diversidad en la enseñanza de las ciencias: Haciauna educación inclusiva y liberadora. Santiago de Chile: Bellaterra, 2017. p. 298.

MEJÍA-CÁCERES, M. A. De las estructuras sociales a los eventos comunicativos: Formación inicial de profesores en ciencias y educación ambiental en el contexto sociopolítico colombiano. [s.l.] Universidade Federal do Rio de Janeiro, 2019.

MOREIRA, A.F.B.; SILVA, T.T da (Org). Currículo Cultura e Sociedade. 2ed.São Paulo: Cortez.1995.

PARRA HEREDIA, J. D. Realismo Crítico: una alternativa en el análisis social. Spcoedad y Economía, v. 31, n. Julio-Diciembre, p. 215-238, 2016. 
RAFFO, R. Lenguaje, comunicación y discurso. $1^{\circ}$ ed. Argentina: Universidad Nacional de Rio Cuarto, 2002.

RAITER, J. Lenguaje y sentido común: las bases para la formación del discurso dominante. Primera ed ed. Buenos Aires, Argentina: Editorial Biblos, 2003.

SORRENTINO, M. et al. Educação ambiental como política pública. Educação e Pesquisa, v. 31, n. 2, p. 285-299, 2005.

TORRES M, Á.; BARRIOS E, A. La enseñanza de las ciencias naturales y educación ambiental en las instituciones educativas oficiales del departamento de Nariño. Tendencias, v. X, n. 1, p. 143-166, 2009.

VAN DIJK, T. A. Semántica del discurso e ideología. Discurso \& Sociedad, v. 21, n. 1, p. 201-261, 2008.

VAN DIJK, T. A. Discurso e cognição na sociedade. Revista Portuguesa de Humanidades, v. 19, n. 1, p. 19-52, 2015.

VAN DIJK, T. A. Análisis Crítico del Discurso. Revista Austral de Ciencias Sociales, n. 30, p. 203-222, 2016. 